Revue européenne des sciences sociales

European Journal of Social Sciences

XLVIII-145 | 2010

À la recherche des fondements de la rationalité

\title{
La Corse vue par les historiens italiens contemporains
}

Giovanni Busino

\section{OpenEdition}

Journals

Édition électronique

URL : http://journals.openedition.org/ress/741

DOI : 10.4000/ress.741

ISSN : 1663-4446

Éditeur

Librairie Droz

Édition imprimée

Date de publication : 1 mars 2010

Pagination : 81-96

ISBN : 978-2-600-01427-4

ISSN : 0048-8046

Référence électronique

Giovanni Busino, "La Corse vue par les historiens italiens contemporains ", Revue européenne des sciences sociales [En ligne], XLVIII-145 | 2010, mis en ligne le 01 mars 2013, consulté le 30 avril 2019. URL : http://journals.openedition.org/ress/741 ; DOI : 10.4000/ress.741 


\section{Giovanni BUSINO}

\section{LA CORSE VUE PAR LES HISTORIENS ITALIENS CONTEMPORAINS}

Les travaux des historiens italiens sur la Corse ainsi que l'inventaire de la documentation archivistique sur cette île ont été enregistrés dans les bibliographies de Renato Giardelli et de Carmine Starace ${ }^{1}$. Presque tous les travaux parus jusqu'à 1910 présupposent que l'Italie d'avant 1860 est une mosaïque d'Etats régionaux aux histoires spécifiques. Pour cette raison les vicissitudes de l'île sont incorporées dans les chroniques et les histoires des Etats dominants ou relatées en tant que relations entre la Corse et l'un ou l'autre Etat du Continent. Les livres remarquables de Giovanni Livi sur les rapports des Corses avec Giovanni et Cosimo De' Medici $^{2}$ ainsi que ses commentaires à l'édition, en 1890, d'une gerbe de lettres inédites de Pascal Paoli en sont les exemples les plus éloquents.

Dès les premières années du $\mathrm{XX}^{\mathrm{e}}$ siècle, à la suite du déclin du positivisme et du tarissement des recherches de l'Ecole historique dite économico-juridique, une nouvelle vision de l'histoire se manifeste, avec force, en Italie. Contre la vision de la spécificité de l'histoire de chaque Etat de la péninsule et contre l'autre que l'histoire de l'Italie commence en 1861 , l'on fait valoir l'unité fondamentale de l'histoire italienne et on la fait remonter tantôt à l'Empire romain tantôt au Haut Moyen-Age. En même temps, la traditionnelle histoire sociale et des institutions est délaissée au profit exclusif de l'histoire politique, de l'histoire de l'Etat, de la Nation et de leur hégémonie sur la société civile.

Les tenants de ce courant historiographique affirment de partager les tribulations et les sentiments de leurs contemporains et dès lors ils revendiquent le droit de récuser la démarcation entre la recherche savante et la divulgation au grand public. Ils sont nationalistes, antisocialistes, antilibéraux, antidémocrates, quelques-uns même antisémites. Tous revendiquent, au nom de la doctrine de l'Irrédentisme, le droit de ramener dans la communauté nationale, entre autre, des territoires désormais français (Nice, Savoie, Corse, Tunisie), anglais (Malte),

1 R. Giardelli, Saggio di una bibliografia generale della Corsica. Prefazione di A. Codignola, Genova, Ed. Giornale storico e letterario della Liguria, 1938, 170 pp. et C. Starace, Bibliografia della Corsica. Presentazione di G. Volpe, Milano, ISPI, 1943, 1033 pp.

2 G. Livi, Delle relazioni dei Corsi colla Repubblica fiorentina e con Giovanni De’ Medici, Firenze, Cellini, 1884, et La Corsica e Cosimo I De’ Medici. Studio storico, Firenze, Boncini, 1885; Lettere inedite di Pasquale d' Paoli, a cura di G.Livi, «Archivio storico italiano », série V, 1890, vol. V, 1, pp. 61 et suiv.; 2, pp. 228 et suiv.; vol. VI, pp. 267 et suiv. Certaines de ces lettres ont été reprises par Franco Venturi in Illuministi italiani. Tomo VII. Riformatori delle antiche repubbliche, dei ducati, dello Stato pontificio e delle isole, a cura di G. Giarrizzo. G. Torcellan et F. Venturi, Milano, Riccardo Ricciardi, 1965, pp. 721-784. 
suisses (le Tessin et les vallées sud alpines des Grisons et des régions romanches). Ces historiens veulent jouer un rôle important dans la bataille pour l'italianité des terres «a redimere», pour un Etat national italien puissant, pour une politique nationaliste et impérialiste ${ }^{3}$.

Les plus représentatifs et les plus actifs dans ce courant à la fois historiographique et politique, sont, entre autres, le romaniste Ettore Pais, l'historien du droit Arrigo Solmi, les modernistes Ettore Rota, Pietro Silva et Francesco Salata. Mais celui qui a exercé l'influence la plus profonde sur des générations de chercheurs c'est assurément Gioacchino Volpe, défini par l'historien Aldo Garosci la «tête de turc de l'historiographie chauvine et impérialiste du régime fasciste $»^{4}$.

Doté d'un talent pugnace et corrosif, d'un savoir historique remarquable, d'une érudition sans faille, d'un remarquable talent pou la saisie des rapports des force, des situations complexes et compliquées et de toutes les forme du «statu nascenti », Volpe, surtout à partir de 1910-1911 environ, abandonne sa spécialisation, les études médiévales, et se consacre aux recherches sur la naissance de l'Etat moderne et la formation de la Nation italienne. L'Etat et la Nation deviennent alors les sujets primordiaux de sa vision de l'histoire, il les décrit comme une force puissante en expansion et en compétition constantes avec les autres Etats et Nations ${ }^{5}$.

Le cas de la Corse lui semble, à ce propos, significatif. Pour lui l'île, placée au cœur de la Méditerranée, a participée depuis toujours à la vie économique, politique et culturelle du système des Etats italiens ${ }^{6}$. Elle a été liée à la République de Pise de 833 à 1340 et puis, sauf les parenthèses Aragonaise, du Saint-Siège et Milanaise (1464-1499), à la République de Gênes dès 1343 et, pour quelques

3 Lire à ce propos B. Bracco, Storici italiani e politica estera : tra Salvemini e Volpe, 1917-1925, Milano, Angeli, 1998; G. Belardelli, Il mito della «Nuova Italia»: Gioacchino Volpe tra guerra e fascismo, Roma, Lavoro, 1988 et surtout G. Volpe, Scritti sul fascismo, 1919-1938, a cura di P. Buscaroli, Roma, Volpe, 1976.

4 Dans «Giustizia e Libertà », V, n. 19, 19 mai 1939 et puis dans A. Garosci, Pensiero politico e storiografia moderne. Saggi di storia contemporanea. I., Pisa, Nistri-Lischi, 1954, p.123 («un retore, che accoppia alle abitudini esteriori della persona colta l'intima disaffezione per la cultura e l'altrettanto intima affezione per il proprio posto.[...] Testa di turco della storiografia sciovinista e imperialista del regime fascista») et p.29 («il male che hanno fatto le intenzioni d'eclettismo equilibrato di costui, per il quale tutti hanno i loro meriti e stanno sullo stesso piano, indipendentemente dalla loro natura di forze rozze e incivili o di forze organizzatrici di civiltà ; esso contiene un elemento molto più demoralizzante che gli eccessi dello storicismo nell'identificare progresso temporale e progresso ideale».

5 Voir G. Volpe, La Nazione e lo Stato italiano, in Il libro d'Italia nel decennale della vittoria, Milano, Istituto fascista di cultura, 1929, mais aussi Piano per una storia d'Italia in collaborazione, désormais in Nel regno di Clio.Nuovi storici e maestri, Roma, Volpe, 1977. Une analyse critique de cette approche se trouve in E. Gentile, La grande Italia. Ascesa e declino del mito della nazione nel ventesimo secolo, Milano, Mondadori, 1997. Cf. pour la problématique générale M. Cuaz, L'identità ambigua: l'idea di «Nazione» tra storiografia e politica, «Rivista storica italiana», CX, fasc.II, agosto 1998, pp. 573-641.

6 Ces thèmes ont été développés de façon fracassante par P. Silva, Il Mediterraneo dall'Unità di Roma all'Impero italiano, Milano, Mondadori, 1927; la $7^{\circledR}$ éd. accrue a été publiée à Milano par 1'ISPI en 1942. Du même voir également Italia fra le grandi potenze (1882-1914), Roma, Cremonese, 1931 et Italia, Francia, Inghilterra nel Mediterraneo, Milano, ISPI, 1939. 
années, au Banco di San Giorgio ${ }^{7}$. L'expédition allemande du comte von Würtemberger en 1731-1732, le royaume de Théodore de Neuhof en 1736-1738-1743, le régime indépendantiste de Pascal Paoli de 1755 à 1768, la présence française dès 1768 ou le royaume anglo-corse entre 1794 et 1796, n'ont pas arraché les racines italiennes de la Corse. La domination génoise, pour certains à la source des révoltes, des frustrations et des injustices, ne lui paraît pas plus oppressive et différente que celles de Naples sur la Sicile et du Piémont sur la Sardaigne. Il affirme que l'équilibre séculaire, linguistique, culturel, politique, économique, a subi par contre, peu à peu, la sape de l'expansionnisme subliminal français. Pour Volpe la France est arrivée à détourner, grâce à des tromperies hypocrites, la Nation corse du processus fusionnant les Etats de la péninsule dans la Nation italienne. Il continue à marteler que la Corse a été italienne jusqu'à 1769, attachée spirituellement à l'Italie de 1860 à 1870 , et qu'elle manifeste toujours son «italianité » dans sa façon d'être et de parler, dans ses idéaux et dans ces comportements culturels. Certes, ajoute-t-il, il ne faut pas confondre l'opinion publique corse favorable à l'Italie avec l'autonomisme et le «corsisme». Ces derniers ne sont que des mouvements minoritaires moralisateurs, passéistes, revendiquant le retour aux modèles et aux règles des anciennes communautés claniques, aux traditions paysannes ancestrales. Ces mouvements alimentent leurs hostilités anti-françaises à la source du rejet du parisianisme, du colonialisme, du parlementarisme, du jacobinisme centralisateur et, en général, de la modernité.

Dans le but de réanimer et ragaillardir l'hégémonie culturelle italienne en Corse, Volpe, avec l'accord et le soutien du philosophe Giovanni Gentile, fonde à Milan, au début de 1924, l'Association «Gli Amici della Corsica» et fait paraître, au mois de mars de cette même année, le premier numéro de la revue "Corsica. Bollettino mensile della Società Gli Amici della Corsica». Les finalités de la Société sont amplement explicitées dans ce premier numéro du «Bollettino» tandis que les articles y font tous l'apologie de l'italianité de l'île.

Le président du Conseil des Ministres, Benito Mussolini écrit, le 25 mai 1924, à Volpe, député au Parlement et professeur à l'Université de Milan, et lui ordonne l'arrêt immédiat du «Bollettino» car sa publication risque de desservir la cause corse, de procurer maints ennuis aux autonomistes et aux amis de l'Italie, de les exposer à une vigilance toujours plus tatillonne de la police française. Volpe obéit sans discuter ${ }^{8}$.

7 Pour la période la plus ancienne de cette «domination» il y a maintenant le travail de G. Petti Balbi, Genova e la Corsica nel Trecento, Roma, Istituto storico italiano per il Medio Evo, 1976.

La lettre a été publiée par E. Di Rienzo, Gioacchino Volpe: fascismo, guerra e dopoguerra. Nuovi documenti, 1924-1945, «Nuova storia contemporanea», VIII, n. 1, gennaio-febbraio 2004, p.122. Sur le fascisme de Volpe voir G. Turi, Il problema Volpe, «Studi storici», XIX, 1978, pp. 175186; G. Belardelli, L'adesione di Gioacchino Volpe al fascismo, «Storia contemporanea», ottobre 1983, pp. 649-694 et G. Sasso, Giovanni Gentile e Gioacchino Volpe dinanzi al crollo del fascismo, in Filosofia e idealismo.IV.Paralipomeni, Napoli, Bibliopolis, 2000, pp.531-557. Les participants au colloque Gioacchino Volpe e la storiografia del Novecento(février-mars 2000) ont essayé d'évaluer l'apport de Volpe à l'organisation des études historiques, au développement de la science historique en Italie et ses engagements politiques pendant le fascisme et après sa chute. Les communications peuvent désormais se lire in «Annali della Fondazione Ugo Spirito», 2000-2001, XII-XIII, pp. 13-221. Mais voir également G. Giarrizzo, Volpe tra storiografia e politica : un bilancio, «L'Acropoli», VII, n. 4, luglio 2006, pp. 411-433 et G. Galasso, Volpe : genesi e senso di 'Italia Moderna', Ibid., pp. 463-482. 
Les articles du «Bollettino» suscitent quelques rares critiques surtout de la part des démocrates libéraux. Par exemple, dans la revue «La rivoluzione liberale» de Piero Gobetti, le journaliste Giovanni Ansaldo dénonce la propagande nationaliste de Volpe, ses accusations malveillantes contre les intellectuels corses, sa façon sournoise de faire de la propagande nationaliste et de dissimuler le dessein, velléitaire et politiquement irréaliste, d'éveiller l'irrédentisme corse?

Indifférent aux critiques et aux mises en garde, Volpe fonde à Milan, en 1925, le «Centro di studi corsi», lance une collection de monographies consacrées à l'histoire de la Corse ainsi que l' «Archivio storico di Corsica», déplacés par la suite à Rome lorsqu'il y sera nommé professeur à la Faculté des sciences politiques de l'Université. Volpe, bien entendu, assume la direction de l' «Archivio» et nomme Ersilio Michel ${ }^{10}$ rédacteur en chef. Ce dernier assurera la direction de la rédaction de la revue jusqu'à sa suppression, en 1943.

Les collaborateurs de l' «Archivio» sont nombreux, la plupart des jeunes de talent, pas tous engagés politiquement. Leurs articles traitent de l'histoire, de la géographie, de l'ethnologie, de la linguistique, de l'archéologie, de l'art, en bref de tous les aspects de la société et de la civilisation corses, souvent avec objectivité et érudition ${ }^{11}$. L' «Archivio» réserve une place importante aux analyses des documents jusqu'alors enfouis dans les archives et les bibliothèques italiennes et étrangères, il publie également des correspondances inédites, des documents érudits, des reconstructions impartiales des événements historiques bien que son directeur ne cache pas sa visée ultime: absorber, assimiler l'histoire de l'île dans celle italienne. Paoli, italien par sa culture, par la langue qu'il parlait et écrivait parfaitement, lui parait un personnage important surtout à cause de la Real-Politik qu'il pratiquait, à cause de son projet de créer un Etat nouveau, de transformer des mentalités primitives, de former une classe dirigeante, de faire reconnaître la Corse au plan international ${ }^{12}$. Toutes ses luttes ambitionnaient, selon Volpe, à donner à l'île un statut international semblable à celui des Etats de la mer Tyrrhénienne et puis à l'intégrer dans l'équilibre politique de l'espace méditerranéen. Les révoltes,de 1729 à 1769, contre Gênes, sont jugées l'œuvre de «irrequieti isolani », intolérants à toute forme de pouvoir. La Convention de Saint-Florent de 1752, le deuxième traité de Compiègne de 1764 et puis le traité de Versailles du 15 mai 1768 prouvent, selon lui, que Gênes n'a jamais renoncé définitivement à

${ }^{9}$ G. Ansaldo, La Corsica, «La Rivoluzione liberale», IV, 1925, 8, p. 33 et Bibliografia della Corsica, Ibid., IV, 1925, 9, p. 39.

${ }^{10}$ Il est l'auteur de nombreux livres et de recherches bien documentées sur les italiens en Egypte, en Tunisie et sur les Esuli italiani in Corsica (1815-1861). Con prefazione di G. Volpe, Bologna, Cappelli, 1940. Voir le portrait qu'en a fait G. Volpe in Storici e maestri. Nuova edizione accresciuta, Firenze, Sansoni, 1967, pp. 198-212.

${ }^{11}$ Les exemples sont nombreux. Il suffit de renvoyer à Gioacchino Volpe e Walter Maturi. Lettere 1926-1961. A cura di Pier Giorgio Zunino, «Annali della Fondazione Luigi Einaudi », XXXIX, 2005, pp. 245-326 et pour une vision d'ensemble à R. De Felice, Gli storici italiani nel periodo fascista, dans son volume Intellettuali di fronte al fascismo. Saggi e note documentarie, Roma, Bonacci, 1985, pp. 190-243.

12 E. Di Rienzo, Lo spazio politico mediterraneo nella storiografia italiana tra Grande Guerra e Fascismo, «Clio», XLII, n. 3, luglio-settembre 2006, pp. 389-400. Du même voir aussi Storia d'Italia e identità nazionale. Dalla Grande Guerra alla Repubblica, Firenze, Le Lettere, 2006, pp. 137-142. 
ses droits souverains. Pour cette raison il qualifie l'annexion de la Corse à la France de forfaiture car la République de Gênes avait le droit de recouvrer sa souveraineté sur l'île après avoir remboursé la dette contractée ainsi que les subsides perçus.

Que la République de Gênes n'aurait jamais pu rembourser des sommes si considérables, ce fait parait à l'historien insignifiant. Désormais Volpe va rendre de plus en plus évanescentes les différences entre la recherche historique et l'idéologie nationaliste au service de la politique étrangère du régime fasciste. Le livre sur la révolte de Sampiero d'Ornano contre la domination génoise et les intrigues de dame Sampiero ${ }^{13}$; celui sur la situation de la Corse à la fin du Moyen-Age ${ }^{14}$ ainsi que l'autre sur les évêques et la question du visiteur apostolique ${ }^{15}$, ont une large diffusion et consolident l'idéologie irrédentiste. Dès les années '30, Volpe peut compter aussi sur l'appui du puissant ISPI ( «Istituto per gli studi di politica internazionale»), de ses ramifications à l'étranger, de ses publications, de collections comme celle intitulée «Interessi e naturali aspirazioni del popolo italiano». Dans toutes les publications de l'ISPI la ésence française à Djibouti et en Tunisie ${ }^{16}$ ainsi que les relations entre l'Italie et la France sont sévèrement critiquées ${ }^{17}$. L'historien Ettore Rota publie des livres colériques et furibonds sur la France «envieuse» de l'Italie, sur la France toujours prête à s'opposer à l'Italie, à en contraster les intérêts légitimes ${ }^{18}$. Paoli est le précurseur du Risorgimento, c'est le premier italien moderne. Etant donné qu'il concevait la liberté de la Corse en tant que partie intégrante de la liberté de l'Italie, les Italiens, selon Volpe et Rota, ont raison de le considérer «le pionnier de leur héroïsme national». Par ailleurs, le

${ }^{13}$ R. Russo, La ribellion di Sampiero Corso, con prefazione di G. Volpe, Livorno, Giusti, 1932 et l'article de I. Rinieri, La vera figura storica di Sampiero Corso. Risposta a «La ribellione di Sampiero Corso» del prof. Rosario Russo, «Archivio storico di Corsica», ottobre-dicembre 1932, pp.461-498.

${ }^{14}$ C. Bornate, Genova e Corsica alla fine del Medio Evo. Prefazione di G. Volpe, Milano, ISPI, 1940.

15 I. Rinieri, I vescovi della Corsica, Livorno, Giusti, 1934.

${ }^{16}$ F. Salata, Il nodo di Gibuti. Storia diplomatica su documenti inediti, Milano, ISPI, 1939; A. Giaccardi, La conquista di Tunisi. Storia diplomatica dal Congresso diu Berlino al trattato del Bardo. Presentazione di F. Salata, Milano, ISPI, 1940; G. Gorrini, Tunisi e Biserta. Memorie storiche. Con prefazione di F. Salata, Milano, ISPI, 1940; R. Catantalupo, Racconti politici dell'altra pace, Milano, ISPI, 1940. Sur les activités de l'institution milanaise, voir. A. Montenegro, Politica estera e organizzazione del consenso. Note sull'Istituto per gli studi di politica internazionale, 1933-1943, «Studi storici», XIX, 1978, pp. 777-817 et E. Decleva, Politica estera, storia, propaganda : l'ISPI di Milano e la Francia (1934-1943), «Storia contemporanea», XIII, n. 4-5, luglio-ottobre 1982, pp. 697 757.

17 Voir le livre d'A. Solmi, Italia e Francia nei problemi attuali della politica europea, Milano, Treves, 1931 et son recueil Discorsi sulla storia d'Italia, con una introduzione, una appendice e note illustrative, Milano, La Nuova Italia, 1941. Cet historien du droit dirigera aussi l' "Archivio storico della Svizzera italiana» de 1926 à 1942. Cette dernière publication inspirera la création de l' «Archivio storico di Malta», dirigé de 1929 à 1934 par Benvenuto Cellini et puis de 1935 à 1942 par Francesco Ercole. Cf.aussi l'apologie de E. Rota, Arrigo Solmi nella sua opera di storico e di politico, Pavia, Treves, 1934.

${ }^{18}$ E. Rota, Italia e Francia dinnanzi alla storia. Il mito della sorella latina, Milano, ISPI, 1939; Francia contro l'Italia dal Risorgimento ad oggi, Milano, Ispi, 1939; Il problema italiano dal 1700 al 1815. L'idea unitaria, Milano, ISPI, 1941; Problemi storici e orientamenti storiografici. Raccolta d'articoli, Como, Cavallero, 1942. 
sort que les Français ont réservé à l'héros corse le prouverait ${ }^{19}$. Cette historiographie, partisane et hagiographique, trouve, en des années tourbillonnantes, beaucoup de supporteurs et des zélateurs fanatiques ${ }^{20}$. Les quatre biographies de Pascal $\mathrm{Paoli}^{21}$, parues à cette même époque, démontrent à quel point l'apologie du fascisme et la défense aveugle de l'idéologie nationaliste ont altéré la recherche en histoire, corrompu les esprits et rendu très compliqués les rapports entre la France et l'Italie. Et pour en avoir des preuves supplémentaires il suffit de parcourir la Storia della Corsica. Dalle origini ai nostri giorni du meilleur spécialiste de l'histoire de l'Ordre de Malte, Mario Monterisi, livre paru en 1939 et puis en $1941^{22}$, ou encore I prodromi della cessione della Corsica : 1727-1789 de Domenico Izzo, publié en $1941^{23}$.

Des revues grand public (par exemple «Corsica antica e moderna», dont le directeur est F. Guerri et le rédacteur en chef l'ex dirigent autonomiste corse Marco Angeli, le «Giornale di politica e di letteratura », directeur Umberto Biscottini, et l'«Almanacco popolare di Corsica») vont diffuser les doctrines nationalistes et impérialistes. Un professeur de l'Université de Pavie, Gino Bottiglioni, publie, à partir de 1933, l' «Atlante linguistico della Corsica», financé par un consortium sous la houlette de l'Université de Cagliari et de différents ministères de Rome.

Le Gouvernement fasciste aide également les «Gruppi di cultura corsi», fondés à Pavie par Petru Giovacchini, nommé «u parrucu». Les 170 sections de ce mouvement, avec environ 22.000 adhérents, confluerons, en 1940, dans l' «Istituto nazionale di studi corsi », dont le siège central est installé à Pavie. La ville de Livourne, où vivaient beaucoup de Corses, est assurément le centre moteur de la propagande irrédentiste. Umberto Biscottini, auteur d'un libelle intitulé «l'île perdue $»^{24}$, en est le concepteur le plus exalté. Il favorise la publication, chez l'Editeur Giusti, de nombreux livres et pamphlets ${ }^{25}$. Le quotidien local, «Il Telegrafo », lance même une édition hebdomadaire corse, financée par le Ministères des affaires étrangères et placée sous la houlette de Francesco Guerri, président également du «Comitato per la Corsica», créé en 1924, comité lequel finance aussi, entre autres, Petru Rocca et son «Partitu corsu d'azzione ${ }^{26}$. De son côté le direc-

${ }^{19}$ E. Rota, Le origini del Risorgimento (1700-1800), Milano, Vallardi, 1938; Pasquale Paoli, Torino, Utet, 1941, spec. p. 147 e p. 194.

${ }^{20}$ Par exemple V. Gayda, Italia e Francia: problemi aperti, Roma, Tip. del Giornale d'Italia, 1939, et Che cosa vuole l'Italia?, Roma,Tip. del Giornale d'Italia, 1940.

${ }^{21}$ L. Ravenna, Pasquale Paoli, con prefazione di N. Rodolico, Firenze, Le Monnier, 1928 (compte rendu de E. Sestan, in «Leonardo», IV, 1928, pp. 271-272); G. Rovida, Pasquale Paoli, Milano, Zucchi, 1940; F. Lancisa, Pasquale Paoli e le guerre d'indipendenza della Corsica, Milano, Vallardi, 1941; E. Rota, Pasquale Paoli, Torino, Utet, 1941.

22 Milano, Bocca, 1939; II éd., Ibid., 1941.

${ }^{23}$ Napoli, Studio di propaganda editoriale, 1941.

${ }^{24}$ U. Biscottini, L'isola persa, Livorno, Giusti, 1930.

${ }^{25}$ Citons, entre autres, L'italianità della Corsica, Livorno, Giusti, 1931; La conquista francese della Corsica. Da un giornale dell'epoca, a cura di F. Guerri, Livorno, Giusti, 1932; P. Monelli, In Corsica, Milano, Garzanti, 1939; A. Pasqualini, Il martirio della Corsica, isola italiana, Firenze, Vallecchi, 1939.

${ }^{26}$ Lire à ce propos F. Guerri, Gli anni e le opere dell'Irredentismo corso. Ricordi nell'ora della redenzione, Livorno, Officine poligrafiche italiane, 1941. 
teur du quotidien, Giovanni Ansaldo, naguère critique virulent de Volpe, fait maintenant l'apologie de l'italianité de l'île, de tout ce que celle-ci doit à Pise et à Gênes. Si la République de Gênes eut été sévère à l'endroit des clans, des bandits et des violents, l'histoire aurait été inévitablement différente: la Corse serait à présent une région italienne, écrit-il ${ }^{27}$. La publication d'un recueil de documents diplomatiques de la République de Vénise est utilisée pour prouver le refus séculaire et opiniâtre des Corses de se soumettre à la domination française ${ }^{28}$. En somme, pour les historiens et les intellectuels fascistes l'Italie sera véritablement grande et indépendante quand les barreaux de la prison méditerranéenne (la Corse, Bizerte, Malte, Suez, Gibraltar) seront détruits, selon les propres mots de Mussolini à la veille de la déclaration de guerre à la France ${ }^{29}$.

Le Gouvernement français, irrité par cette propagande importune et inamicale, après quelques protestations diplomatiques sans résultats, interdit la diffusion, sur son territoire, en 1928, du quotidien «Il Telegrafo», et en 1932 de l' «Archivio» ainsi que des publications historiques et politiques revendiquant l'«italianité » de la Corse $^{30}$. Cependant la propagande à outrance ne s'arrête point. En 1936 Petru Giovacchini commence à organiser, dans presque toutes les régions italiennes, les «Gruppi di cultura corsa».

Les écrits de Volpe sur la Corse, parus entre 1923 et 1940, sont republiés, revus, modifiés, puis réunis en volumes, accompagnés ou suivis par des innombrables préfaces aux publications de ses élèves ou de ses collaborateurs. Les idées du maître sont amplifiées par les articles de certains élèves et vont constituer, pendant presque une vingtaine d'année, les fondements d'un véritable paradigme historiographique. Les plus importants de ces écrits se trouvent, à présent, réunis dans le volume Storia della Corsica italiana ${ }^{31}$, puis résumés par l'auteur dans l'article Corsica du Dizionario di politica ${ }^{32}$, édité par Antonino Pagliaro pour le compte du Partito nazionale fascista.

${ }^{27}$ Les articles d'arguments corses de ce journaliste ont été réunis dans le volume: G. Ansaldo, Corsica l'isola persa, Genova, De Ferrari, 1999. Dans ce recueil se trouvent des véritables perles, comme celle qu'on peut voir à la p. 182: «La parola malgoverno genovese non regge, ha le sue radici nella razza e nel sangue».

${ }^{28}$ B. Brunelli, Corsi contro Francesi nei dispacci dei consoli veneti, Milano, ISPI, 1941.

${ }^{29}$ Lire à ce propos pour d'autres d'informations: R. De Felice, Mussolini il Duce. Lo Stato totalitario, 1936-1940, Torino, Einaudi, 1996. Le marquis Blasco Lanza d'Ajeta était en charge, à Rome, du suivi de la politique fasciste en Corse. Sa biographie est encore à écrire.

${ }^{30}$ Voir les Documents diplomatiques français, 1932-1939, $2^{\mathrm{e}}$ série, tome XIII, Paris, Imprimerie Nationale, 1979, n. 354, p. 635; tome XIV, 1980, n. 130,p. 229; tome XVII, 1984, n. 329, p. 542; tome XVIII, 1985, n. 380, p. 453.

${ }^{31}$ G. Volpe, Storia della Corsica italiana, Milano, ISPI, 1939. Récemment S. Tomassini, Gli studi sulla Corsica in «Annali della Fondazione Ugo Spirito », op.cit., pp. 755-87, a proposé une nouvelle lecture des écrits de Volpe sur la Corse. Les réticences à l'égard de certaines de ses prises de position sont nombreuses ainsi que de la conclusione, où on lit: Volpe «al di là di qualsiasi illusione, oggi e da tempo ormai davvero impossibile, ci ha insegnato almeno due cose: la prima, generale, che le nazion i hanno vita come di person e, possono cresc ere e ridursi, possono morire e a volte perfino nascere eo c redere di esser nate - che è poi la stessa cosa-, la seconda, specifica per la Corsica, che i francesi posson o tranquillamente riconoscersi il 'diritto di non essere indifferenti'».

${ }^{32}$ Roma, Istituto dell'Enciclopedia italiana, 1940, I, pp. 650-656. 
La vision historique de Volpe en tant qu'histoire de l'Etat, est jugée, dans la revue «La critica», en 1923 et en 1939, par Benedetto Croce, mais les écrits sur l'histoire corse sont ignorés ${ }^{33}$. Le seul à les analyser sévèrement est un jeune, Franco Venturi ${ }^{34}$, dont la renommée de très grand historien ne fera que croître avec les années, soit en Europe soit aux Etats-Unis d'Amérique qu'en Russie.

Pour Venturi l'histoire de Volpe et de ses disciples est «une histoire fantaisiste», élaborée uniquement pour les usages de la politique du fascisme. Faire du massacre de Pontenuovo la première bataille du Risorgimento italien revient à écrire une histoire imaginaire ${ }^{35}$. La défaite de Paoli et des Corses par les troupes françaises, le 9 mai 1769, marque plutôt la fin d'une glorieuse indépendance et le commencement des tribulations d'un peuple héroïque et indomptable. D'après Venturi la vision historique de Volpe et des ses consorts déforme «l'importance de la révolte corse du XVIII ${ }^{\mathrm{e}}$ siècle», «sa valeur propre, originale dans l'intransigeance de sa haine anti-génoise». Si l'Europe des Lumières s'enthousiasma pour la rébellion des Corses, si Rousseau, Boswell, Parini ou Alfieri, si des nombreux intellectuels européens et une frange de colons nord-américains purent la voir comme le modèle ou le symbole du progrès et de la modernité, c'est parce qu'il s'agissait de la rébellion «des pauvres contre les riches, des pures aborigènes contre les étrangers corrupteurs, du petit nombre énergique contre le faible grand nombre, des paysans contre les citadins». Pour Venturi «Il est par conséquent ridicule de voir les érudits fascistes tâcher de mettre des voiles pudiques sur l'origine même de la révolte, et faire tous leurs efforts pour démontrer que l'administration génoise n'était pas si mauvaise qu'on l'a dit, que les Corses n'étaient pas malheureux qu'on l'a prétendu sous la domination des banquiers sérénissimes.» En ignorant l'origine anti-génoise de l'indépendance corse, les «érudits fascistes»s'interdisent «la compréhension non seulement de Paoli et des siens, mais aussi de cette légende corse que le dix-huitième siècle tissa autour du courage et de la fierté insulaires ». Les «érudits fascistes» dissimulent «les accusations de sépulcre blanchi que Paoli adressait à l'Italie de son temps, incapable de sentir assez l'originalité et l'importance de son effort», ils ignorent la différence «qui passe entre la première et la seconde partie de la vie de Paoli, [et] ils confondent son premier généralat avec ses intrigues anglaises pendant la révolution française ». «Sa froideur, son habilité s'illumine d'un idéal de raison. Il parle bien de mâter les révoltes intérieures, les séditions continuelles [...], mais cela ne reste pas un but, n'est pas la fin ultime de la politique.» Et Venturi rappelle que cet idéal est le point cardinal de Paoli, lequel écrit dans une de ses plus belles lettres: «Je me flatte qu'un jour l'esprit de sédition finira; les guerres civiles guérissent les préjugés des nations et quand elles sont finies les rendent plus respectables et

${ }^{33}$ L. Del Piano, Gioacchino Volpe e la Corsica ed altri saggi, Cagliari, Cuec, 1987.

${ }^{34}$ Gianfranchi, Le fascisme contre Paoli, «Fascisme et Italie. Bimensuel français de 'Giustizia e Libertà'», V, n. 48, 9 décembre 1938, p. 4. Il y a deux traductions italiennes de ce texte, la première in F. Venturi, La lotta per la libertà. Scritti politici. Saggi introduttivi di V. Foa e A. Galante Garrone. A cura di L. Casalino, Torino, Einaudi, 1996, pp. 127-134, et la deuxième in F. Venturi, Pagine repubblicane. A cura di M. Albertone e con un saggio introduttivo di B. Baczko, Torino, Einaudi, 2004, pp. 217-222. Dans le bimensuel du 16 décembre est annoncé une conférence de Venturi à Strasbourg sur le thème «Paoli, la Corse et le fascisme».

${ }^{35}$ Lire l'article de G. Volpe, Pontenuovo, «La Tribuna», 11 maggio 1923. 
le gouvernement devient plus fort. A cette vérité doivent la plus grande partie de leurs victoires Louis XIV, Cromwell et Guillaume III.» Pour Venturi Paoli «est encore trop vivant pour rester tout entier dans les mains de Volpe et autres académiciens ».

A la suite des bouleversements de 1943 l' «Archivio», le «Centro di studi corsi » ainsi que l'appareil de la propagande irrédentiste sont démantelés. La défaite du régime fasciste et la fondation de la République ne poussent pas Volpe à repenser son historiographie. Il continuera à professer la doctrine de l'hégémonie de l'Etat-Nation italien, de ses droits impérialistes en Méditerranée, qu'il exposera minutieusement dans les trois volumes de son œuvre majeure Italia moderna et dans un de ses derniers articles sur l' «italianité» de la Corse paru en $1964^{36}$. Certes, il doit reconnaître l'échec en Corse du «nouveau irrédentisme » et pourtant il reste convaincu qu'il faut continuer à défendre les droits italiens en Méditerranée afin d'éviter leur prescription et afin de ne pas barrer pour de bon «la via alle possibilità dell'avvenire ${ }^{37}$.

L'histoire des années 1940-1943 reste un terrain vierge. On dispose, pour le moment, du volume des mémoires du général italien commandant les troupes d'occupation ${ }^{38}$ ainsi que des documents de l'Etat majeur de l'Armée italienne publiés en 1990 et d'une reconstruction historique des rapports entre le gouvernement de Pétain et celui de Mussolini ${ }^{39}$.

Les archives, par exemple, du Ministère de la culture et des Affaires étrangères nous apportent beaucoup d'informations sur les avatars de la propagande italienne au service de la politique irrédentiste du régime fasciste et de son expansionnisme territorial ${ }^{40}$. D'autres informations pourraient nous venir des archives françaises.

${ }^{36}$ Voir à ce propos son dernier texte à propos de la Corse: G. Volpe, Il nazionalismo tra le due guerre, «Il Veltro», VIII, n.3, giugno 1964, pp. 481-504. A propos de l'historiographie de Volpe, cf. G. di Giovanni, Il realismo storico di Gioacchino Volpe, Roma, Semerano, 1964 et les articles réunis de AA.VV. par R. Bonuglia in Gioacchino Volpe tra passato e presente, Roma, Aracne Editrice, 2007 et les livres cités plus haut.

${ }^{37}$ Cf. G. Volpe, Lettere dall'Italia perduta, a cura di G. Belardelli, Palermo, Sellerio, 2006 et les analyses de G. Sasso, Guerra civile e storiografia, «La cultura», XLIII, 2005, n. 1, pp. 5-25; G. Santomassimo, Dopoguerra storiografico e astratti furori, «Passato e Presente», n. 64, gennaio-aprile 2005, pp. 157-167 ainsi que de P. G. Zunino, Il «fascismo degli idealisti». Alla caduta del regime: Gioacchino Volpe e le radici della politica estera fascista, «Annali della Fondazione Luigi Einaudi », XL, 2006, pp. 3-41. Eugenio Di Rienzo vient de publier une biographie exhaustive de Volpe fort bien documentée. A propos des dernières années de la vie Volpe, voir la IV $\mathrm{IV}^{\mathrm{e}}$ partie de ce livre La storia e l'azione. Vita di Gioacchino Volpe, Firenze, Le Lettere, 2008, intitulée «Straniero in Patria». Sur le la genèsi du nationalisme de Volpe lire du même Di Rienzo, Ancora su liberalismo, liberismo e fascismo. I gruppi nazionali liberali nel 1919, «L'Acropoli», VII, n. 4, luglio 2006, pp. 434-462, texte repris avec des changements mineurs dans la biographie citée.

${ }^{38}$ G. Magli. Le truppe italiane in Corsica prima e dopo l'armistizio del 1943, Lecce, Tipografia della Scuola A.U.C., 1950.

39 R.H. Rainero, Mussolini e Petain. Storia dei rapporti tra l'Italia e la Francia di Vichy (10 giugno 1940-8 settembre 1943). Stato maggiore dell'Esercito. Ufficio storico, Roma, Marzorati, 1990, 2 vols. et M. Borgogni, Mussolini e la Francia di Vichy. Dalla dichiarazione di guerra al fallimento del riavvicinamento italo-francese (giugno 1940-aprile 1942), Siena, Nuova Immagine, 1991.

${ }^{40}$ Lire à ce propos A. Giglioli, Il fascismo e la questione dell'Irredentismo corso, « Nuova Antologia », fasc. 2212, ottobre-dicembre 1999, pp. 331-357. 
Entre 1944 et 1950 pas de publications, en Italie, sur la Corse mais les idées de Volpe continuent à circuler et on les retrouve même, atténuées ou simplement suggérées, dans des travaux d'histoire économique, où le traité du 16 mai 1768, ratifié par la République de Gênes le 28 du même mois, est qualifié «una beffa» et Paoli désigné comme l' «anticipatore del Risorgimento», le champion de l'indépendance nationale italienne ${ }^{41}$.

Il faudra attendre encore quelques années avant d'assister à la floraison des «études élégantes», selon la belle formule de Benedetto Croce. En 1952 paraît une recherche bien documentée sur la politique méditerranéenne anglaise pendant la guerre de succession d'Autriche ${ }^{42}$ et en 1954 la reconstruction, en vérité sans souffle, de la médiation milanaise à l'occasion du différend de 1746 entre Gênes et l'Autriche ${ }^{43}$.

Quelques années auparavant Ernesto Sestan proposait une thèse interprétative nouvelle à propos des rapports de la Corse avec Gênes ${ }^{44}$. Pour cet historien le féodalisme fut importé par Gênes sur l'île alors qu'il y était totalement incompatible à cause de la nature non hiérarchique, très segmentée de la structure sociale prééxistante. Or ce système féodale importé a exacerbé les Corses, les a poussé à s'enfermer dans des clans, à pratiquer des formes agressives de socialité, à employer la violence pour la solution des conflits, la vendetta pour laver les torts subis, à percevoir l'étranger comme un ennemi et n'importe quelle forme de domination comme une tyrannie pernicieuse. Pour Sestan Paoli eut le génie de comprendre que la solution du problème corse exigeait la formation non de la Nation corse mais de l'Etat corse reconnus et garanti dans son indépendance par les grandes puissances européenne, un Etat indépendant organisé à partir des traditions et des volontés de ses habitants, en mesure de faire valoir tant soit peu l'intérêt général et le bien public. L'administration de la justice constitue le cœur de sa construction étatique. Certes, Paoli voulait que l'italien fût la langue de cet Etat, mais rien ne prouve qu'il acceptât de rattacher l'Etat corse aux Etats de la péninsule et plus tard de le dissoudre dans celui du Royaume d'Italie ${ }^{45}$.

${ }^{41}$ V. Vitale, Breviario della storia di Genova. Lineamenti storici ed orientamenti bibliografici, Genova, Società ligure di storia patria, 1955, pp. 365-416, mais également G. Giacchero, Storia economica del Settecento genovese, Genova, Apuania, 1951; Economia e società del Settecento genovese, Genova, Sagep, 1973; Seicento e le compere di San Giorgio, Genova, Sagep, 1979.

${ }^{42}$ C. Baudi di Vesme, La politica mediterranea inglese nelle relazioni degli inviati italiani a Londra durante la cosidetta «Guerra di successione d'Austria», 1741-1748, Torino, Gheroni, 1952.

${ }^{43}$ A. Ostoja, Genova nel 1746: Una mediazione milanese nelle trattative austro-genovesi, Bologna, Palmaverde, 1954.

${ }^{44}$ E. Sestan, Sardegna e Corsica e Nazione italiana, in Europa settecentesca ed altri saggi, Milano, Ricciardi, 1951, pp. 243-263. Dans Scritti vari. III. Storiografia dell'Otto e Novecento, a cura di G. Pinto, Firenze, Le Lettere, 1991, pp.355-360, Sestan affirme que 1'historiographie de Volpe est fondée su «nessi impensati fra motivi apparentemente affini, immersi e fusi in un fluido omogeneo».

${ }^{45}$ E. Sestan, op. cit., pp.256-257: «...Pasquale Paoli, per quanto con la volubile ambiguità inerente al termine potesse parlare di nazione corsa, non mirò à formare una nazione corsa, non almeno nell'accezione moderna del termine, ma a formare uno stato corso, riconosciuto e garantito nella sua indipendenza dalle grandi potenze europee; e precisamente uno stato corso che si aggiungesse, indipendente, su un medesimo piano, agli altri stati italiani indipendenti, non uno stato corso che si ponesse fuori dell'orbita della comune nazione morale e culturale italiana, non una nazione equidistante dalla nazione italiana, e dalla nazione francese e dalle altre nazioni. Il pnsiero indipendentista, 
Toute la problématique de ce domaine d'étude est bouleversée lorsque Franco Venturi commence à publier ses travaux sur Paoli et la Corse à partir des années ' $60^{46}$. Tout d'abord ces travaux approfondissent et prolongent la critique à l'historiographie nationaliste entreprise à la fin des années '30 avec l'article Le fascisme contre Paoli et ensuite ils ouvrent des perspectives nouvelles à propos de la spécificité, de l'originalité du «Settecento riformatore » raccordé davantage à la Philosophie des Lumières qu'aux péripéties du Risorgimento ${ }^{47}$.

Il a été écrit que Venturi affiche une trop grande sympathie pour Paoli car l'homme lui parait incarner les luttes pour la liberté et pour l'égalité, car Paoli est à la fois un modernisateur, un réformateur social, un critique impitoyable de la civilisation corrompue, car il est l'exemple vivant de l'unité entre la pensée et l'action. C'est vrai mais il y a plus. Pour l'historien italien Paoli a été capable de relier le sentiment national au cosmopolitisme, de combiner les avantages d'un gouvernement éclairé avec les aspirations égalitaires et libertaires du peuple, mais aussi de conjurer les mythes révolutionnaires et nationalistes et de concevoir la Nation, la Patrie et la Liberté en tant que des biens présents et futurs, comme la source originaire de l'espérance des générations présentes et de celles à venir ${ }^{48}$.

Venturi interprète, peut-être avec un certain anachronisme, l'insurrection corse en tant que révolte coloniale contre un Etat patricien, en tant que combat contre les privilèges d'une aristocratie citadine décadente et survivant grâce à une exploitation des plus cyniques. Toutefois il a bien vu combien réaliste et pourtant proche des idéaux et des problèmes de la philosophie des Lumières était le projet de Paoli, ancré dans les attentes des Corses de vivre en hommes libres, égaux et

non nazionalista corso del Paoli riecheggia ancora...». Une vision plus lisse est celle de F. Valsecchi, L'Italia del Settecento, Milano, Mondadori, 1971, pp. 191-200.

${ }^{46}$ F. Venturi, Pasquale Paoli, in Illuministi italiani. Tomo VII. Riformatori delle antiche repubbliche, dei ducati, dello Stato pontificio e delle isole, a cura di G.Giarrizzo, G. Torcellan e F. Venturi, Milano-Napoli, Riccardo Ricciardi, 1965, pp. 719-784; Genova a metà del Settecento, «Rivista storica Italiana », LXXIX, fasc.4, dicembre 1967, pp. 1129-1143; L'Italia fuori d'Italia, in Storia d'Italia. Volume terzo. Dal primo Settecento all'Unità, Torino, Einaudi, 1973, pp. 1049-1052; Pasquale Paoli e la rivoluzione di Corsica, «Rivista storica italiana», LXXXVI, fasc. 1, marzo 1974, pp. 5-81; Il dibattito francese e britannico sullarivoluzione di Corsica, Ibid., fasc.4, dicembre 1974, pp. 643-719; Il dibattito in Italia sulla rivoluzione di Corsica, Ibid., LXXXVIII, fasc.1, marzo 1976, pp. 40-89; Pasquale Paoli in Olanda, in Hommages à Fernand Ettori, «Etudes corses», X, 1982, nn. 18-19, pp. 191-200; Patria e libertà : la rivoluzione di Corsica, in Settecento riformatore. V.L'Italia dei Lumi (1764-1700). Tomo primo. La rivoluzione di Corsica. Le grandi carestie degli anni sessanta. La Lombardia delle riforme, Torino, Einaudi, 1987, pp. 3-220

${ }^{47}$ En ce sens aussi L. Guerci, Gli studi venturiani sull'Italia del '700: dal «Vasco» agli illuministi, in Il coraggio della Ragione. Franco Venturi in tellettuale e storico cosmopolita, a cura di L. Guerci e G. Ricuperati, Torino, Fondazione Luigi Einaudi, 1998, pp. 203-241.

48 Voir à ce propos E. Tartarolo, La rivolta e le riforme. Appunti per una biografia intellettuale di Franco Venturi (1914-1994), «Studi Settecenteschi», 15, 1995, pp. 9-42 et également R. Pasta, Franco Venturi e le antiche repubbliche italiane, in Il repubblicanesimo moderno. L'idea di repubblica nella riflessione di Franco Venturi, a cura di M. Albertone, Napoli, Bibliopolis, 2006, pp. 379406; G. Cambiano, L'illuminismo di Franco Venturi, Ibid., pp. 125-127 et B. Baczko, Curiosité historique et passions républicaines, Ibid., pp. 217-247; G. Imbruglia, Illuminismo e storicismo nella storiografia italiana, Napoli, Bibliopolis, 2003, pp. 257-393 et pp. 341-478; D. Grippa, Il percorso di un'intransigenza: Franco Venturi e gli scritti del 1945-1946, «Annali della Fondazione Luigi Einaudi », XXXVII, 2003, pp. 447-495. 
indépendants. La politique à l'endroit de la religion, de la justice indépendante et le réajustement de la tactique militaire au profit de la guerre des partisans sont décrits en tant que des manifestations significatives de cette façon moderne de gouverner. Venturi souligne avec force que Paoli, pour concilier la tradition et les réformes, pour dépasser les affrontements claniques, pour changer les mentalités traditionnelles, pour élaborer une volonté collective, comprit qu'il fallait, sans plus tarder, construire un Etat, le doter d'une administration, d'une armée, d'une justice, d'une Université capable de former une classe dirigeante aux valeurs communes. Et il s'attela à cette tâche de construire l'Etat corse, de le structurer démocratiquement, d'en faire le garant des libertés collectives et individuelles, avec la patience, la prudence et la sagesse de l'homme politique de génie et du philosophe cosmopolite ${ }^{49}$.

Venturi est plus réservé sur le comportement de Paoli pendant les deux exiles anglais ${ }^{50}$, sur l'analyse que le général faisait de la domination française jugée, à tort, pareille à celle de Gênes. L'exil ternit, selon l'historien italien, ses capacités d'intuitions, ses analyses du nouveau patriotisme en train de surgir et ses jugements sur la nouvelle conjoncture politique créée par la Révolution de 1789. La venue à Paris, son discours du 22 avril 1790 à l'Assemblée constituante, son retour en Corse révèlent l'irréalisme de son agir politique. Il pensait de pouvoir braver la Convention et le Comité de salut public de la même manière qu'il l'avait fait avec Louis XV et ses généraux. Or nonobstant les avertissements, les attaques et les injures des Jacobins, de Buonarroti et de Bonaparte, Paoli donna la Corse à la Couronne britannique en 1794 sans jamais avoir la conscience du fait que le Gouvernement anglais, peu intéressé à une colonisation directe ou indirecte, se débarrasserait de lui et de la Corse à la première occasion. Ce que le Gouvernement de Sa Majesté fit effectivement en 1796 tout en acceptant d'accueillir à nouveau, en Angleterre, Pascal Paoli et quelques-uns de ses partisans ${ }^{51}$.

Venturi réserve aux dernières années de la biographie de Paoli des pages d'une empathie attendrissante. Elles décrivent la passion avec laquelle Paoli suivait les vicissitudes de l'Université de Corté, sa création choyée, l'institution à laquelle il avait confié la mission de pacifier les esprits, de faire progresser la civilisation en Corse et dans l'Europe désormais sous le joug de Napoléon. Mais ces pages mettent aussi en évidence le décalage entre la vision politique de Paoli et la nouvelle réalité internationale très défavorable à la cause de l'indépendance de l'île. La lecture des pages intitulées «Patrie et liberté: la révolution de Corse» nous révèlent non seulement la culture encyclopédique de l'historien cosmopolite mais aussi son admiration pour Paoli le réformateur fidèle aux doctrines des

\footnotetext{
${ }^{49}$ Sur cette question voir D. Carrington, L'ordinamento costituzionale della Corsica durante il regime di Pasquale Paoli, «Critica storica». XI, n.s., n. 4, dicembre 1974, pp. 594-622.

${ }^{50}$ Lire à ce propos C. Vivanti, Lettere di Pasquale Paoli dall'Inghilterra, «Rivista storica italiana», LXXI, fasc. I, aprile 1950, pp. 88-118. Sur la base des 13 lettres à Giambattista Gherardo d'Arco, Vivanti avance l'hypothèse que la pensée politique de Paoli est plus proche du libéralisme modéré que du républicanisme.

${ }^{51}$ Malgré les interventions des supporteurs de la Corse et leurs efforts pour convaincre le gouvernement de Sa Majesté des avantages commerciaux à tirer d'une présence sur l'île, les autorités anglaises furent inébranlables. Voir à ce propos M. Ambrosoli, John Symonds. Agricoltura e politica in Corsica e in Italia (1765-1770), Torino, Fondazione Luigi Einaudi, 1974, pp. 37-59.
} 
Lumières. Elles nous éclairent sur ce que Paoli fit en Corse, sans aides extérieures, entre 1755 et 1768, mais aussi pourquoi aucun Etat ne soutint ni protégea les luttes des insulaires et pourquoi, en même temps, elles devinrent partout la plus belle des épopées du XVIII ${ }^{\mathrm{e}}$ siècle et plus tard un véritable mythe romantique.

L'historien turinois reconstruit, sur la base d'une documentation étendue et souvent inédite, de quelle façon les «affaires corses» furent reçues, perçues ou transfigurées en Piémont, en Lombardie, à Venise, en Toscane, dans les Etats de l'Eglise et à Naples ${ }^{52}$. Et il nous rapporte comment ces mêmes réceptions, perception et transfiguration naissent et se développent en France, en Angleterre, en Hollande, dans les Cantons suisses, dans le monde germanique, dans la Russie de Catherine II et chez les colons d'Amérique du Nord. Partout Paoli y est vu comme l'incarnation des idéaux réformistes, modernisateurs, progressistes, comme le modèle de l'homme d'Etat philosophe. Ces pages nous apprennent, en outre, de quelle façon les idées circulaient à l'époque des Lumière et ajoutent des informations sur le «Projet de constitution pour la Corse» de Rousseau, sur le chapitre «De la Corse» du Précis du siècle de Louis XV de Voltaire, sur les attente et le critiques à propos des affaires corses du réformateur piémontais Dalmazzo Francesco Vasco ${ }^{53}$ ainsi que sur les agissements et les intrigues, dès 1768, de Mathieu Buttafoco, chef du parti royaliste pendant la Révolution française et dont la correspondance avec Paoli sur la Constitution de la Corse a été publiée, en Sardaigne, en $1978^{54}$.

B. Brunelli Bonetti a pu reconstruire les péripéties italiennes et les velléités du roi Théodore ${ }^{55}$ tandis que $\mathrm{H}$. Benedikt, sur la base des relations des diplomates autrichiens et de lettres inédites de Paoli, nous a donné des renseignements très précieux sur les combats des Corses pour la liberté et l'indépendance ${ }^{56}$.

${ }^{52}$ Quelques années auparavant ce chantier avait été ouvert par C. Bordini, Note sulla fortuna di Pasquale Paoli nel Settecento italiano, «Rassegna storica del Risorgimento», 60, 1973, fasc. 4, pp. 522-543, article puis repris dans le livre du même Rivoluzione corsa e illuminismo italiano, Roma, Bulzoni, 1979. Cet auteur est de l'avis que les réformateurs lombards ont défendu le droit des Corses à se rebeller contre le despotisme de la Dominante mais qu'ils ne croyaient pas que les luttes de Paoli constituaient une expérience exemplaire. C'est en Toscane que les Corses ont trouvé des alliés et des appuis plus sûrs. Le pape Clément XIII était indifférent aux conflits opposant les Génois aux Corses car pour lui la défense des droits de l'Eglise contre l'élargissement du pouvoir juridictionnel des Etats primait sur tout. A Naples, le ministre Bernardo Tanucci regardait aux événements corses avec méfiance. Sur un certain nombre de points les interprétations de Bordin se veulent plus détachées et froides que celles de Venturi. Sur la politique de Clement XIII, pape de 1758 à 1769, défenseur des Jésuites et contempteur de l'esprit philosophique, lire F. Fonzi, Le relazioni fra Genova e Roma al tempo di Clemente XIII, «Annuario dell'Istituto storico italiano per l'età moderna e contemporanea », vol. VIII, 1957. pp. 7-196.

${ }^{53}$ Sur ce réformateur piémontais il faut consulter également le livre de F. Venturi, Dalmazzo Francesco Vasco (1732-1794), Paris, Droz, 1940 et les références à Paoli se trouvent in D.F. Vasco, Opere, a cura di S. Rota Ghibaudi, Torino, Fondazione Luigi Einaudi, 1966, passim.

${ }^{54}$ Voir leurs échanges à propos de la Constitution in Rousseau et la Corsica nel carteggio di Pasquale Paoli \& M. Buttafuoco, a cura di G. Deroyer et G. Rais, Cagliari, Edizioni 3T, 1978.

${ }_{55}$ B.Brunelli Bonetti, Teodoro re di Corsica,in Miscellanea in onore di Roberto Cessi, Roma, Edizioni di storia e letteratura, 1958, II, pp. 453-467.

${ }^{56} \mathrm{H}$. Benedikt, La lotta di liberazione dei Corsi secondo le relazioni delle Legazioni austriache e lettere inedite di Pasquale Paoli, in Miscellanea in onore di Roberto Cessi, cit., II, pp. 435-46. 
Dès les années '70 les historiens italiens ont recommencé à labourer le terrain de la recherche érudite.

Enrica Dardani a publié un faisceau de documents, de la période 1759-1768, lesquels éclairent les instructions imparties par Guillaume-Léon Du Tillot, le tout puissant ministre du duc Don Philippe, à comte d'Argental, plénipotentiaire du Duc auprès du roi de France, à propos d'un éventuel rattachement de la Corse au Duché de Parme ${ }^{57}$.

Les historiens génois contemporains et notamment Carlo Bitossi, à partir des années '90, nous ont donné les premières études d'histoire économique génoise solides et perspicaces et grâce auxquelles une bonne partie des polémiques et supputations sur les rapports entre la République de Gênes et la Corse commencent désormais à être éclaircies ${ }^{58}$.

Francesco Barra a analysé les rapports économiques et politiques entre la Corse et le Midi de l'Italie de 1754 à 1815 et sur l'émigration corse à Naples ${ }^{59}$ tandis que Marco Cini a remis sur le métier les investigations sur les rapports culturels entre l'Île et la Péninsule et nous a révélé des informations très précises à propos d'une matière ayant suscité par le passé des affrontements polémiques et des mises en cause des identités de deux peuples ${ }^{60}$.

Alessandra Giglioli a publié, en 2001, la reconstruction des relations entre l'Italie e la France sur la base d'un examen approfondi de toute la documentation du Ministère italien des affaires étrangères et de celle actuellement consultable à Paris dans les archives du Quai d'Orsay ${ }^{61}$. Ce livre décrit par le menu l'organisation que le gouvernement fasciste mit en place (en Corse, en France et en Italie)

${ }^{57}$ E. Dardani, La Corsica nel carteggio du Tillot-d'Argental dell'Archivio di Stato Parmense, in «Studi e ricerche della Facoltà di economia e commercio dell'Università degli Studi di Parma», 14, 1978, pp. 435-451.

58 Voir à ce propos A. Ceccarelli, Dieci anni di studi sull'antico regime genovese (1528-1797), «Rivista storica italiana », CXIX, fasc.II, agosto 2007, pp. 727-777 ainsi que C. Bitossi, Il governo dei magnifici. Patriziato e politica a Genova fra Cinque e Seicento, Genova, Ecig, 1990; «La repubblica è vecchia». Patriziato e governo a Genova nel secondo Settecento, Roma, Istituto storico italiano per l'età moderna e contemporanea, 1995; Oligarchi. Otto studi sul ceto dirigente della Repubblica di Genova (XVI-XVII secolo), Genova, Dipartimento di storia moderna e contemporanea, 1995; L'antico regime genovese, 1576-1797, in Storia di Genova, a cura di D. Puncuh, Genova, Società ligure di storia patria, 2003, pp. 301-509.

${ }^{59}$ F. Barra, La Corsica e il Mezzogiorno d'Italia tra Settecento borbonico e decennio napoleonico (1734-1815), in Corsica. Isola problema tra Europa e Mediterraneo, a cura di A. Aversano, Napoli, ESI, 1998, pp. 25-44.

${ }^{60}$ La nascita di un mito: Pasquale Paoli tra '700 e '800, a cura di M. Cini, Pisa, BFS, 1998; M. Cini, Le dialogues des élites. Salvatore Vitale-Giovan Pietro Viesseux: Correspondance (18291847), Ajaccio, Albiana, 1999; M. Cini, Gli esuli italiani in Corsica. 1815-1860, in Storia, letteratura, linguistica, a cura di A. Bocchi e M. Cini, Pisa, Domus Mazziniana, 2000; M. Cini, Storia nazionale e costruzione identitaria: le «novelle storiche» corse, in «Etudes Corses», n. 55, décembre 2002, pp. 107-125; M. Cini, Une île entre Paris et Florence: culture et politique de l'élite corse dans la première moitié du XIX siècle, Ajaccio, Albiana, 2003. Sur la question de l'identité la bibliographie est considérable. Ici il suffit de renvoyer à G. Bollati, L'Italiano. Il carattere nazionale come storia e come invenzione, Torino, Einaudi, 1983 et à A. Volpi, Appunti sulla storia d'Italia nella cultura europea dell'Ottocento, in «Etudes corses», n. 55, décembre 2002, pp. 23-46.

${ }^{61}$ A. Giglioli, Italia e Francia 1936-1939. Irredentismo e ultranazionalismo nella politica estera di Mussolini, Roma, Jouvence,2001. 
pour propager l'Irrédentisme, les montants des subsides versés à la presse corse et française, des subventions et des dons attribués aux «collaborateurs». L'auteur nous donne un exposé détaillé et complet du rôle du quotidien «Il Telegrafo» et des tentatives des Autorités françaises d'en surcontrer l'influence soit en interdisant sa diffusion dans l'Hexagone soit en demandant à Rome la suppression de l'édition hebdomadaire corse. Les officiels fascistes étaient bien conscients, notamment dès 1938, que la propagande irrédentiste n'avait aucune chance de succès, qu'elle produisait surtout la détérioration des relations diplomatiques entre les deux pays, et pourtant ils continuaient à croire en la nécessité d'une annexion de la Corse pour assurer et garantir l'hégémonie italienne sur les mers méditerranéennes.

Ce livre de Madame Giglioli explore et décrit sereinement les anfractuosités d'un passé piteux; il met en évidence les velléités d'une politique expansionniste et inévitablement condamnée à la faillite ${ }^{62}$. L'approche des rapports italo-français, pendant la période 1936-1939, sous l'angle des revendications territoriales italiennes fait conclure à son auteur que le «coup de poignard» du mois de juin 1940, n'est pas « un gesto incomprensibile e inatteso »; il est plutôt la lame de fond « di contrasti e di problemi ancora vivi e aperti. [...] la politica di Mussolini verso la Francia non aveva fatto altro che coltivare e aggravare questo dissidio, utilizzando a tal fine tutte le risorse disponibili messe a disposizione sia dalla diplomazia internazionale, sia dall'efficiente propaganda del regime.» Madame Giglioli affirme que «il motivo delle rivendicazioni territoriali italiane verso la Francia non rappresenta soltanto una chiave di lettura per comprendere la politica estera fascista negli anni precedenti lo scoppio della seconda guerra mondiale, ma costituisce la 'sostanza' stessa di tale politica, motivo essenziale dell'entrata in guerra dell'Italia, obiettivo prioritario perseguito con fermezza e intransigenza fino alla disfatta del paese e al crollo del regime.» ${ }^{63}$

Il est évident qu'une telle explication découle de l'analyse de la politique étrangère fasciste centrée exclusivement sur les revendications territoriales italiennes et sur les obstacles et les difficultés opposés par la France à ces revendications. Elle ne tient pas compte du système stratégique mis en place par les alliances entre les puissances de l'Asse et les griefs italiens contre le Traité de Versailles, contre le fonctionnement du parlementarisme et contre la démocratie. Pour cette raison l'explication proposée dans l'excellent et fort bien documenté livre de Madame Giglioli peut paraître unilatérale à certains historiens du $\mathrm{XX}^{\mathrm{e}}$ siècle.

En 2003 paraît, enfin, le premier volume (La prise du pouvoir) contenant les lettres de Paoli de la période 1749-1756, suivi en 2005 du deuxième (La construction de l'Etat) couvrant les années 1756-1758, en 2007 du troisième (Le visiteur

${ }^{62}$ Op. cit., p. 635 : «Il caso corso è ancora una volta esemplare. In Corsica, infatti, l'azione irredentistica si svolse principalmente tramite agenti e elementi fiduciari locali, i quali si rivelarono ben presto interessati più alla possibilità d'estorcere denaro dalle borse del governo fascista, che a sostenere la causa dell'italianità dell' isola. La stessa situazione, [...], caratterizzò l'affare dei sovvenzionamenti alle destre francesi, in particolare quelli a Doriot.[...] La proclamazione delle mire italiane sulla Corsica determinò infatti la reazione degli autonomisti e propagandisti corsi, molti dei quali ruppero con l'Italia, causando il fallimento del tentativo di creare un movimento autonomista autonomo.»

${ }^{63}$ Op. cit., p. 637. 
apostolique) contenant les lettres de années 1759-1760 et du quatrième (L'avenir de la Corse est sur l'eau), à paraître, avec les lettres de la période 1761-1762. Les volumes sont enrichis d'un apparat critique magistral et de remarquables introductions, celles de F. Ettori, de J.-M. Arrighi, de C. Bitossi et de A.-M. Graziani. Ces volumes nous font déjà entrevoir l'importance exceptionnelle de ce joyau littéraire qu'est la correspondance de Pascal Paoli pour la connaissance de l'histoire de la Corse et du XVIII ${ }^{\mathrm{e}}$ siècle européen. On peut aisément prévoir que cette correspondance va relancer aussi les études corses en Italie, qu'elle aidera les historiens italiens à concevoir autrement les relations entre les deux pays, à ne plus enfreindre, comme il est arrivé au cours de la première moitié du $\mathrm{XX}^{\mathrm{e}}$ siècle, les règles morales de la recherche, ces règles morales à propos desquelles Bronislaw Baczko nous a enseigné: quand l'historien les enfreint, «il est en proie à la mauvaise conscience et aux sentiments d'avoir renié et trahi ses devoirs moraux ainsi que le rôle social qu'il aurait dû assumer ${ }^{64}$.

L'historiographie italienne d'aujourd'hui semble échapper à une telle calamité: ayant perdu l'aveugle certitude de la compréhension idéologique du passé, la hantise de ce que l'on n'a pa connu ou vécu, ayant désormais un rapport distant au passé et à la tyrannie du présent, en s'efforçant maintenant de reconstruire la chaîne des raisons qui permettent de relier, de façon intelligible, le présent à ce qui a disparu à jamais et nous est devenu étranger, cette historiographie qui ne confond plus mémoire et histoire est en train de conquérir une conscience historique et de la mémoire collective à la fois plus sereine et complexe. Elle prépare ainsi l'éclosion de la nouvelle saison des études historiques sur les relations de l'Italie avec l'Île de Beauté.

giovanni.busino@bluewin.ch $61-70$

${ }^{64}$ B. Baczko, La responsabilité morale de l'historien, «Diogène », n. 67, juillet-octobre 1969, pp. 